\title{
Disease Damage Influences Cardiovascular Risk Reclassification Based on Carotid Ultrasound in Patients with Systemic Lupus Erythematosus
}

\author{
Juan C. Quevedo-Abeledo, Íñigo Rúa-Figueroa, Hiurma Sánchez-Pérez, Beatriz Tejera-Segura, \\ Antonia de Vera-González, Alejandra González-Delgado, Javier Llorca, \\ Miguel Á. González-Gay, and Iván Ferraz-Amaro
}

\begin{abstract}
Objective. Composite scores of cardiovascular (CV) risk factors underestimate the CV risk in patients with systemic lupus erythematosus (SLE). Carotid artery ultrasound (US) was found useful in identifying high CV-risk patients with inflammatory arthritis. We assessed the effect of carotid US assessments on the CV risk stratification of patients with SLE.

Methods. This cross-sectional study included 276 patients with SLE. These indices were measured: lipid profile, Systematic COronary Risk Evaluation (SCORE) risk calculation, and disease activity (SLE Disease Activity Index), severity (Katz), and damage [Systemic Lupus International Collaborating Clinics (SLICC)/American College of Rheumatology Damage Index]. Carotid plaques were assessed by US. A multivariable regression analysis, adjusted for classic CV-related factors, was performed to evaluate how risk reclassification was influenced by disease characteristics in patients with SLE.

Results. Thirty-six percent of patients had carotid plaques. However, only $6 \%$ of them fulfilled the definitions for high or very high risk according to the SCORE risk charts. Following carotid US assessment, $32 \%$ of the patients were reclassified as very high risk. Disease duration (OR 1.04, 95\% CI $1.00-1.07, \mathrm{p}=0.025)$ and a SLICC $>0$ (OR $2.4895 \%$ CI 1.15-5.34, p = 0.020) were independently associated with a higher risk of reclassification. A predictive model for reclassification included age (cutoff 52 yrs, sensitivity 60\%, specificity 86\%), disease duration (cutoff 24 yrs, sensitivity $40 \%$, specificity $82 \%$ ), presence of hypertension, SLICC $>0$, waist circumference (cutoff $102 \mathrm{~cm}$, sensitivity $48 \%$, specificity $84 \%$ ), and C3 (cutoff $127 \mathrm{mg} / \mathrm{dl}$, sensitivity $52 \%$, specificity $92 \%$ ) and triglyceride (cutoff $140 \mathrm{mg} / \mathrm{dl}$, sensitivity 68\%, specificity 79\%) serum levels.

Conclusion. Reclassification into a very high-risk category is frequent after carotid US assessments in patients with SLE. This is independently influenced by disease damage. (First Release January 15 2019; J Rheumatol 2019;46:483-91; doi:10.3899/jrheum.180881)
\end{abstract}

Key Indexing Terms:

SYSTEMIC LUPUS ERYTHEMATOSUS

CAROTID PLAQUES

CARDIOVASCULAR RISK SCORE

From the Division of Rheumatology, Hospital Doctor Negrín, Las Palmas de Gran Canaria; Division of Rheumatology, Hospital Universitario de Canarias, Tenerife; Division of Rheumatology, Hospital Insular, Las Palmas de Gran Canaria; Central Laboratory Division, Hospital Universitario de Canarias, Tenerife; Department of Epidemiology and Computational Biology, School of Medicine, University of Cantabria; CIBER Epidemiología y Salud Pública (CIBERESP), Instituto de Investigacion Sanitaria (IDIVAL); Division of Rheumatology, Hospital Universitario Marqués de Valdecilla, IDIVAL; Epidemiology, Genetics and Atherosclerosis Research Group on Systemic Inflammatory Diseases, Hospital Universitario Marqués de Valdecilla, IDIVAL; School of

Medicine, University of Cantabria, Santander, Spain; Cardiovascular Pathophysiology and Genomics Research Unit, School of Physiology, Faculty of Health Sciences, University of the Witwatersrand, Johannesburg, South Africa.

This work was supported by a grant to IFA from the Spanish Ministry of Health, Subdirección General de Evaluación y Fomento de la Investigación, Plan Estatal de Investigación Científica y Técnica y de Innovación 2013-2016 and by Fondo Europeo de Desarrollo Regional FEDER - (Fondo de Investigaciones Sanitarias, FIS PI14/00394,

PI17/00083). Prof. González-Gay's research was supported by European
Union FEDER funds and by the Fondo de Investigación Sanitaria (grants PI06/0024, PS09/00748, PI12/00060 and PI15/00525) of the Instituto de Salud Carlos III (ISCIII, Health Ministry, Spain). It was also partially supported by RETICS Programs RD12/0009 (RIER), RD12/0009/0013, and RD16/0012 from the Instituto de Salud Carlos III (ISCIII, Health Ministry, Spain).
J.C. Quevedo-Abeledo, MD, Division of Rheumatology, Hospital Doctor Negrín; I. Rúa-Figueroa, MD, PhD, Division of Rheumatology, Hospital Doctor Negrin; H. Sánchez-Pérez, MD, Division of Rheumatology, Hospital Universitario de Canarias; B. Tejera-Segura, MD, Division of Rheumatology, Hospital Insular; A. de Vera-González, MD, Central Laboratory Division, Hospital Universitario de Canarias;
A. González-Delgado, MD, Central Laboratory Division, Hospital Universitario de Canarias; J. Llorca, MD, PhD, Department of Epidemiology and Computational Biology, School of Medicine, University of Cantabria, and CIBERESP, IDIVAL; M.A. González-Gay, MD, PhD, Division of Rheumatology, Epidemiology, Genetics and Atherosclerosis Research Group on Systemic Inflammatory Diseases, Hospital Universitario Marqués de Valdecilla, IDIVAL, and School of Medicine, University of Cantabria, and Cardiovascular Pathophysiology and Genomics Research Unit, School of Physiology, Faculty of Health

Personal non-commercial use only. The Journal of Rheumatology Copyright @ 2019 . All rights reserved. 
Systemic lupus erythematosus (SLE) is associated with an increased and premature prevalence of atherosclerosis ${ }^{1}$. This probably stems from the compound effects of a genetic component, classic cardiovascular (CV) risk factors, disease severity, and the therapy used to manage the disease $e^{2,3,4,5}$. Although new drugs have substantially contributed to longer survival rates, it has become evident that $\mathrm{CV}$ disease has emerged as one of the most important causes of morbidity and mortality in these patients ${ }^{6}$. A systematic review has revealed that the risk of $\mathrm{CV}$ disease is 5 times as high in patients with SLE as in the general population ${ }^{7}$. Moreover, in young women with SLE, the age-specific incidence of CV disease is higher by a factor of as much as $50^{8}$.

All current guidelines on the prevention of $\mathrm{CV}$ disease in clinical practice recommend an assessment of total $\mathrm{CV}$ risk because atherosclerosis is usually the product of a number of risk factors. Composite scores, such as the Framingham Risk Score $^{9}$ and the Systematic COronary Risk Evaluation $(\mathrm{SCORE})^{10}$, have been used to predict longterm CV risk in the general population. This is important because prevention of CV disease in an individual should be tailored to his or her $\mathrm{CV}$ risk: the higher the risk, the more intense the action undertaken should be ${ }^{11}$. Nevertheless, when applied to patients with SLE, these classic scores have been found to significantly underestimate the true risk of $\mathrm{CV}$ disease ${ }^{12,13}$. This inadequate stratification of the CV risk is an issue of major importance in patients with SLE, and the traditional approach does not entail significant differences in the management of risk factors ${ }^{14}$. Thus, the search for additional tools that could identify high CV-risk patients with SLE who may benefit from active therapy to prevent $\mathrm{CV}$ events is of major importance.

Carotid artery US were found useful in identifying high $\mathrm{CV}$-risk patients with rheumatoid arthritis (RA) who fulfilled the definitions for moderate $\mathrm{CV}$ risk according to well-established risk charts ${ }^{15,16}$. Therefore, screening for asymptomatic atherosclerotic plaques by carotid ultrasound (US) should be regarded as part of the $\mathrm{CV}$ disease risk evaluation in patients with RA and other forms of inflammatory joint disorders according to European League Against Rheumatism (EULAR) recommendations ${ }^{17}$. Because of the clinical consequences related to an indication for statin treatment if carotid plaques are present, we wondered whether this procedure could be of additional value to $\mathrm{CV}$ risk stratification in patients with SLE.

Taking all these considerations into account, the main

Sciences, University of the Witwatersrand; I. Ferraz-Amaro, MD, PhD, Division of Rheumatology, Hospital Universitario de Canarias.

Drs. M.A. González-Gay and I. Ferraz-Amaro shared senior authorship. Address correspondence to Dr. I. Ferraz-Amaro, Division of Rheumatology, Hospital Universitario de Canarias, 38320 Santa Cruz de Tenerife, Spain.E-mail:iferrazamaro@hotmail.com, miguelaggay@hotmail.com

Accepted for publication September 21, 2018. purpose of our study was to assess the effect of carotid US assessments on the CV risk stratification of patients with SLE who were initially assessed by SCORE risk charts. We also aimed to identify patient characteristics that could potentially predict such $\mathrm{CV}$ risk reclassifications.

\section{MATERIALS AND METHODS}

Study participants. This was a cross-sectional study that included 276 patients with SLE. All of them were 18 years old or older and were already enrolled when they fulfilled $\geq 4$ American College of Rheumatology (ACR) 1997 classification criteria for SLE ${ }^{18}$. They had been diagnosed by rheumatologists and were periodically followed up at rheumatology outpatient clinics. For the purpose of inclusion in our present study, SLE disease duration needed to be $\geq 1$ year. Patients with SLE undergoing biologic therapy (belimumab or rituximab) were not excluded from our present study. Likewise, because glucocorticoids are often used in the management of SLE, patients taking prednisone were not excluded. None of the patients had established CV disease. However, patients were excluded if they had a history of cancer or any other chronic disease, evidence of active infection or a glomerular filtration rate $<60 \mathrm{ml} / \mathrm{min} / 1.73 \mathrm{~m}^{2}$. The study protocol was approved by the Institutional Review Committee at Hospital Universitario de Canarias and Hospital Doctor Negrín, both in Spain, and all subjects provided informed written consent (approval no. 2015-84).

Assessments and data collection. Surveys in patients with SLE were performed to assess CV risk factors and medication. Subjects completed a questionnaire and underwent a physical examination to determine anthropometric measurements and blood pressure. Medical records were reviewed to ascertain specific diagnoses and medications. Hypertension (HTN) was defined as a systolic or diastolic blood pressure $>140 \mathrm{mmHg}$ and $90 \mathrm{mmHg}$, respectively. Dyslipidemia was defined if 1 of the following factors was present: total cholesterol $>200 \mathrm{mg} / \mathrm{dl}$, triglyceride $(\mathrm{TGC})>150 \mathrm{mg} / \mathrm{dl}$, high-density lipoprotein (HDL) cholesterol $<40$ in men or $<50 \mathrm{mg} / \mathrm{dl}$ in women, or low-density lipoprotein (LDL) cholesterol $>130 \mathrm{mg} / \mathrm{dl}$. Atherogenic index was calculated using the total cholesterol/HDL cholesterol ratio. SLE disease activity and damage were assessed using the SLE Disease Activity Index (SLEDAI-2K) ${ }^{19}$ and the Systemic Lupus International Collaborating Clinics (SLICC)/ACR Damage Index (SDI) ${ }^{20}$, respectively. For the purpose of our present study, the SLEDAI index was split into none (0), mild (1-5), moderate (6-10), and high and very high activity $(>10)$ as previously described ${ }^{21}$. Disease severity was measured as well, using the Katz Index ${ }^{22}$. Additionally, standard techniques were used to measure plasma glucose, $\mathrm{C}$-reactive protein (CRP), and serum lipids.

Carotid ultrasound assessment. Carotid ultrasound was performed to assess carotid intima-media wall thickness (cIMT) in the common carotid artery and to detect focal plaques in the extracranial carotid tree in patients with SLE. A commercially available scanner, Mylab 70 (Esaote), equipped with a 7-12 MHz linear transducer and an automated software-guided radiofrequency technique - Quality Intima Media Thickness in real-time (QIMT, Esaote) - was used for this purpose. Based on the Mannheim consensus, plaque criteria in the accessible extracranial carotid tree (common carotid artery, bulb, and internal carotid artery) were defined as follows: a focal protrusion in the lumen measuring at least cIMT $>1.5 \mathrm{~mm}$; a protrusion at least $50 \%$ greater than the surrounding cIMT; or an arterial lumen encroaching $>0.5 \mathrm{~mm}^{23}$.

Statistical analysis. Demographic and clinical characteristics were described in patients with SLE as mean \pm SD or percentages for categorical variables. For non-normally distributed continuous variables, data were expressed as median and interquartile range (IQR). Univariate differences between reclassified and non-reclassified patients were assessed through Student t, Mann-Whitney U, chi-square, or Fisher's exact tests according to normal distribution or number of subjects. Logistic regression analysis adjusted for the variables with a $\mathrm{p}$ value $<0.20$ in the univariate analysis was performed to assess the relation of SLE disease-related data with the presence of reclas-

Personal non-commercial use only. The Journal of Rheumatology Copyright (C) 2019. All rights reserved 
sification. An all-sets logistic regression model was constructed to describe the most parsimonious combination of predictors of risk reclassification according to Akaike Information Criteria, Schwarz Bayesian Criterion, the area under the curve, and Hosmer-Lemeshow goodness-of-fit. For characteristics that were associated with reclassification and that were included in the predictive model, sensitivity versus false positive frequency (1-specificity) was analyzed utilizing receiver-operating characteristic curves. To determine the optimal cutoff value of baseline characteristics in predicting reclassification, we calculated the Youden index using the following formula: sensitivity + specificity -1 , with the maximum obtained value corresponding to the optimal cutoff point. All the analyses used a 5\% two-sided significance level and were performed using SPSS software, version 21 (IBM), and STATA software, version 15/SE (Stata Corp.). A p value $<0.05$ was considered statistically significant.

\section{RESULTS}

Demographic, analytical, and disease-related data. A total of 276 SLE patients with a mean \pm SD age of $51 \pm 12$ years were included in this study. Demographic and disease-related characteristics of the participants are shown in Table 1. Body mass index (BMI) was $27.49 \pm 5.7 \mathrm{~kg} / \mathrm{m}^{2}$ and the average waist circumference was $92 \pm 13 \mathrm{~cm}$. Traditional CV risk factors were frequent. Specifically, $40 \%$ and $38 \%$ were hypertensive or taking antihypertensive therapy, respectively. In addition, $68 \%$ of the patients had dyslipidemia and $25 \%$ were current smokers. Seventy-five $(27 \%)$ of the patients were on statins. Laboratory assessments disclosed CRP of 1.90 (IQR 0.90-4.90) $\mathrm{mg} / \mathrm{l}$ and a total cholesterol of $194 \pm 39 \mathrm{mg} / \mathrm{dl}$. LDL and HDL cholesterol were $107 \pm 33$ and $62 \pm 20 \mathrm{mg} / \mathrm{dl}$, respectively.

The median SLE disease duration was $18 \pm 10$ years and the SLICC and Katz indices were 1 (IQR 0-2) and 2 (IQR $1-4)$, respectively. One hundred six $(38 \%)$ of the patients were categorized as having no activity (i.e., in remission)

Table 1. Demographic data of the 276 patients with SLE.

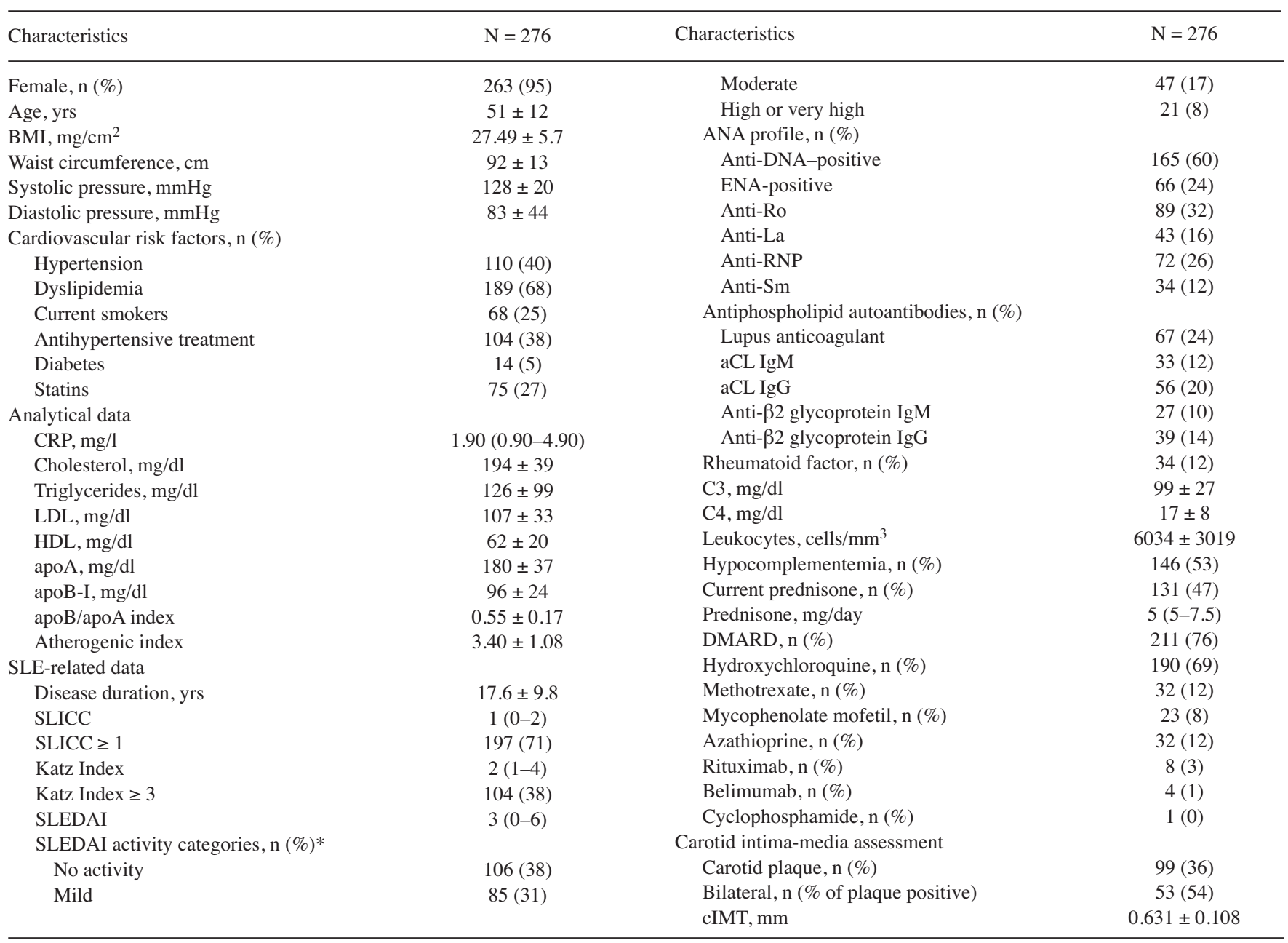

Values are mean \pm SD or median (IQR) when data were not normally distributed. * SLEDAI categories were defined as follows: 0 (no activity); 1-5 (mild); 6-10 (moderate); > 10 (high or very high). SLE: systemic lupus erythematosus; BMI: body mass index; CRP: C-reactive protein; LDL: low-density lipoprotein; DMARD: disease-modifying antirheumatic drug; aCL: anticardiolipin antibodies; HDL: high-density lipoprotein; apoA: apolipoprotein A; ANA: antinuclear antibodies; ENA: extractible nuclear antibodies; SLEDAI: SLE Disease Activity Index; SLICC: Systemic Lupus International Collaborating Clinics/American College of Rheumatology Damage Index; cIMT, carotid intima-media thickness; IQR: interquartile range. 
based on the SLEDAI-2K index, while $31 \%, 17 \%$, and $8 \%$ were included in the mild, moderate, and high or very high categories, respectively. Almost a half of them (47\%) were taking prednisone [5 (IQR 5-7.5) $\mathrm{mg} /$ day]. One hundred sixty-five patients $(60 \%)$ were found to be positive for anti-DNA, and $62(24 \%)$ expressed some of the extractible nuclear antibodies (ENA) at the time of the study. One hundred ninety patients (69\%) were taking hydroxychloroquine, while mycophenolate mofetil (MMF), azathioprine, rituximab, and belimumab were less frequently used. Additional disease-related information is shown in Table 1.

Regarding carotid US assessment, $36 \%$ of the patients had carotid plaques. The average cIMT was $0.631 \pm 0.108 \mathrm{~mm}$.

SCORE risk category reclassification after carotid sonography. Following SCORE risk chart stratification, 184 (67\%) and 73 (27\%) patients were included in the low and moderate $\mathrm{CV}$ risk categories, respectively (Table 2). Only 16 patients $(6 \%)$ fulfilled the definitions for high or very high CV risk. However, carotid US assessment resulted in 32\% patients being reclassified as very high $\mathrm{CV}$ risk. In this regard, 43 (23\%) of the 184 included in the category of low $\mathrm{CV}$ risk, based on the SCORE risk charts, had carotid plaques. They were therefore reclassified as very high CVrisk patients. As was described in patients with $\mathrm{RA}^{15,16}$, the use of carotid US yielded more relevant results in those patients with SLE included in the category of moderate CV risk. This was because, according to the SCORE, 43 of 73 patients (59\%) had carotid plaques, and consequently had to be reclassified as very high $\mathrm{CV}$-risk (Table 2).

Differences between reclassified and non-reclassified patients into very high $C V$-risk categories after carotid US. Differences in recorded characteristics between patients who were reclassified following the carotid US assessment and those who were not reclassified were observed (Table 3 ). While patients were older $(57 \pm 9$ vs $48 \pm 11$ yrs, $\mathrm{p}<0.001)$ and HTN more common (56 vs $31 \%, \mathrm{p}<0.001)$ in the reclassified cohort, sex, BMI, waist circumference, and the presence of dyslipidemia, current smoking, or diabetes did not reveal any differences between the 2 groups. In addition, cIMT was higher in those patients who were reclassified $(0.671 \pm 0.121$ vs $0.615 \pm 0.097 \mathrm{~mm}, \mathrm{p}<0.001)$. Interestingly, none of the laboratory data related to lipid profiles or
CRP showed any difference between reclassified and non-reclassified patients.

Regarding SLE-related features, some differences were also noted. Disease duration ( $16 \pm 9$ vs $21 \pm 11$ yrs, $\mathrm{p}<0.001)$ was found to be higher in the reclassified patients. Similarly, SLICC, both as a continuous ( $\log$ SLICC: $1.04 \pm 0.60$ vs 0.70 $\pm 0.62, \mathrm{p}<0.001$ ) and categorical (SLICC >0: 86 vs $64 \%$, $\mathrm{p}<0.001$ ) variable, was found to be higher in the reclassified patients. Differences were still apparent even when this index was constructed in a manner that excluded those items related to $\mathrm{CV}$ disease. However, Katz and SLEDAI indices, anti-DNA positivity, and the presence of ENA or antiphospholipid antibodies did not show any differences between reclassified and non-reclassified individuals. Only C3, anti-RNP positivity, and the use of MMF revealed some differences (Table 3).

Multivariable regression analysis confirmed the aforementioned results. Disease duration showed some correlation with reclassification after adjusting for age, HTN, waist circumference, diabetes, and TGC (OR 1.04, 95\% CI $1.00-1.07, p=0.025)$. Similarly, an SLICC higher or equal to 1 (OR 2.48, 95\% CI 1.15-5.34, p = 0.020) and log SLICC (OR $1.63,95 \%$ CI $1.01-2.64, p=0.045)$ showed a statistically significant relationship to reclassification after adjusting for age and CV risk factors. These relationships were also found when the SLICC was used without the CV-related factors (SLICC CV-), although in the case of log SLICC it was marginally significant (OR $1.56,95 \%$ CI $0.95-2.64$, $\mathrm{p}=0.077)$.

Predictive model for reclassifying patients into the high CVrisk category following a carotid US assessment. A predictive model was constructed only for patients with SLE in the low-risk SCORE category. These variables conjointly represented the most parsimonious model capable of predicting reclassification of patients with SLE into the very high CVrisk category (Table 4): age, disease duration, HTN, an SLICC CV > 0, C3 serum levels, abdominal circumference, and TGC. Moreover, age older than 52 years, a disease duration longer than 24 years, a waist circumference $>127$ $\mathrm{cm}$, and C3 complement and TGC > $127 \mathrm{mg} / \mathrm{dl}$ and 140 $\mathrm{mg} / \mathrm{dl}$, respectively, were the cutoffs among the continuous variables that reached the highest Youden indices.

Table 2. Reclassification of patients with SLE following carotid ultrasound.

\begin{tabular}{|c|c|c|c|c|c|}
\hline \multirow{2}{*}{\multicolumn{2}{|c|}{ Initial SCORE Risk Category }} & \multicolumn{4}{|c|}{ Cardiovascular Risk Category after Carotid Ultrasound Assessment } \\
\hline & & Low & Moderate & High & Very High \\
\hline Low & 184 & 141 & 0 & 0 & 43 \\
\hline Moderate & 73 & 0 & 30 & 0 & 43 \\
\hline High & 9 & 0 & 0 & 2 & 7 \\
\hline \multirow[t]{2}{*}{ Very high } & 7 & 0 & 0 & 0 & 7 \\
\hline & 273 & 141 & 30 & 2 & 100 \\
\hline
\end{tabular}

Three patients of 276 were excluded from the analysis because reclassification data were not available. SLE: systemic lupus erythematosus; SCORE: Systematic COronary Risk Evaluation. 
Table 3. Differences between reclassified and non-reclassified patients with SLE into the very high CV risk category following carotid ultrasound assessment.

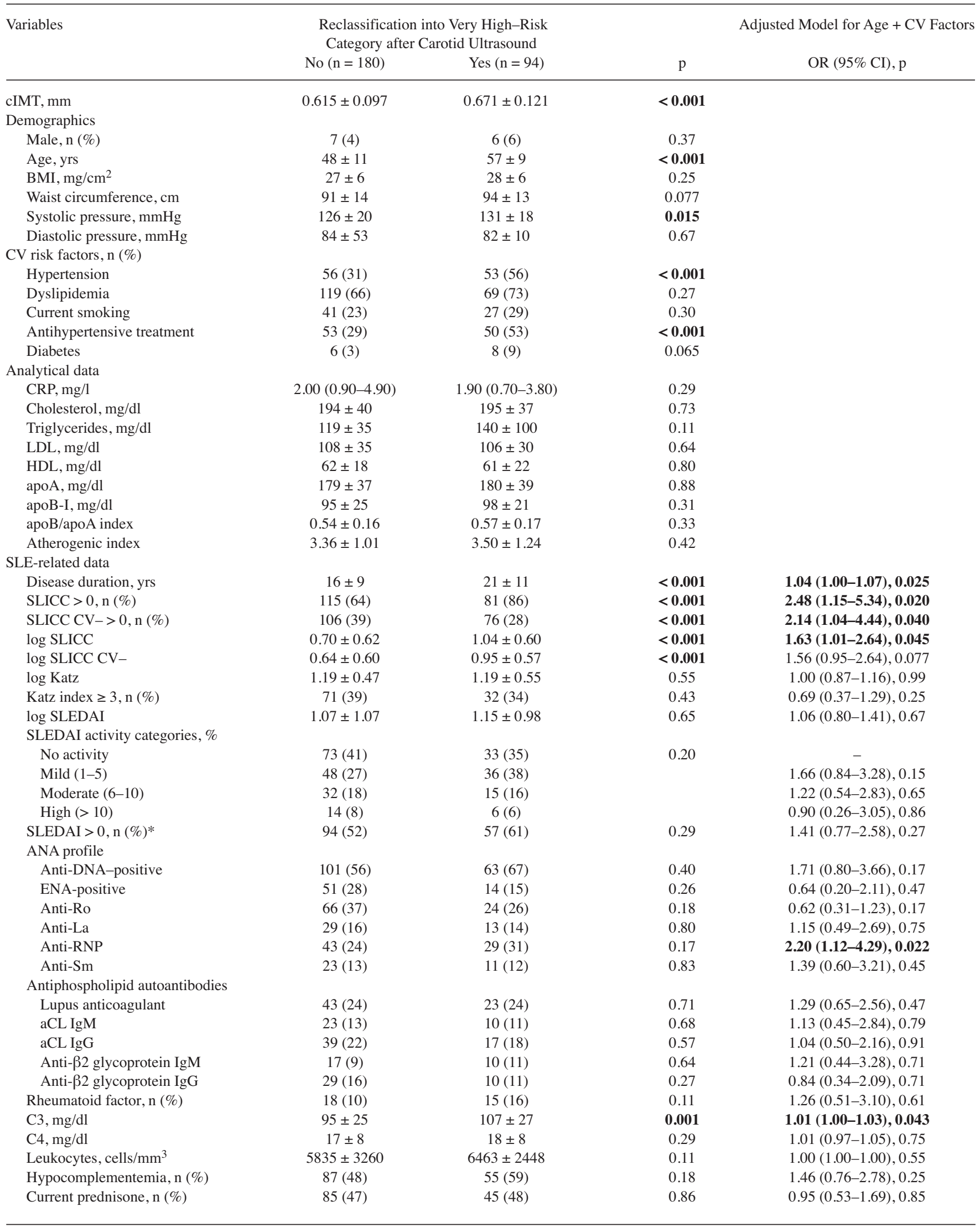


Table 3. Continued.

\begin{tabular}{|c|c|c|c|c|}
\hline \multirow[t]{2}{*}{ Variables } & \multicolumn{2}{|c|}{$\begin{array}{l}\text { Reclassification into Very High-Risk } \\
\text { Category after Carotid Ultrasound }\end{array}$} & \multicolumn{2}{|c|}{ Adjusted Model for Age + CV Factors } \\
\hline & No $(n=180)$ & Yes $(n=94)$ & $\mathrm{p}$ & OR $(95 \% \mathrm{CI}), \mathrm{p}$ \\
\hline DMARD, $\mathrm{n}(\%)$ & $138(77)$ & $72(77)$ & 0.89 & $1.40(0.71-2.77), 0.33$ \\
\hline Hydroxychloroquine, n (\%) & $128(71)$ & $61(65)$ & 0.34 & $1.02(0.55-1.89), 0.95$ \\
\hline Methotrexate, n (\%) & $17(9)$ & $15(16)$ & 0.11 & $1.33(0.57-3.10), 0.51$ \\
\hline Rituximab, n (\%) & $5(3)$ & $2(2)$ & 0.75 & $1.13(0.19-6.73), 0.89$ \\
\hline Belimumab, n (\%) & $3(2)$ & $1(1)$ & 0.69 & - \\
\hline Cyclophosphamide, n (\%) & $0(0)$ & $1(1)$ & 0.078 & - \\
\hline
\end{tabular}

Values in bold face are statistically significant. Adjusted variables were age, hypertension (binary variable), waist circumference, diabetes, and triglycerides. Two patients' reclassification information was not available $(\mathrm{n}=274)$. Data represent mean $\pm \mathrm{SD}$ or median $(\mathrm{IQR})$ when data were not normally distributed. * SLEDAI categories were defined as follows: 0 (no activity); 1-5 (mild); 6-10 moderate; > 10 (high or very high). SLE: systemic lupus erythematosus; CV: cardiovascular; cIMT: carotid intima-media wall thickness; BMI: body mass index; CRP: C-reactive protein; LDL: low-density lipoprotein; DMARD: disease-modifying antirheumatic drug; aCL: anticardiolipin antibodies; HDL: high-density lipoprotein; ANA: antinuclear antibodies; ENA: extractible nuclear antibodies; SLEDAI: SLE Disease Activity Index; SLICC: Systemic Lupus International Collaborating Clinics/American College of Rheumatology Damage Index; SLICC CV- : SLICC calculated without CV items; apoA: apolipoprotein A; IQR: interquartile range.

Table 4. All subset logistic regression models for the prediction of reclassification in patients with SLE.

\begin{tabular}{|c|c|c|c|c|c|}
\hline Variables & OR $(95 \% \mathrm{CI})$ & $\mathrm{p}$ & Optimal Cutoff & Sensitivity, $\%$ & Specificity, $\%$ \\
\hline Age, yrs & $1.15(1.06-1.24)$ & 0.001 & 51.9 & 60 & 86 \\
\hline Disease duration, yrs & $0.99(0.94-1.05)$ & 0.78 & 23.9 & 40 & 82 \\
\hline SLICC CV $->0$ & $1.07(0.82-1.40)$ & 0.76 & & & \\
\hline $\mathrm{C} 3, \mathrm{mg} / \mathrm{dl}$ & $1.01(1.00-1.04)$ & 0.033 & 127 & 52 & 92 \\
\hline Abdominal circumference, $\mathrm{cm}$ & $0.98(0.95-1.02)$ & 0.37 & 102 & 48 & 84 \\
\hline AIC & 114.7 & & & & \\
\hline $\mathrm{BIC}$ & 137.5 & & & & \\
\hline AUC & 0.852 & & & & \\
\hline Sensitivity & 44.4 & & & & \\
\hline Specificity & 97.0 & & & & \\
\hline pfitHL & 0.118 & & & & \\
\hline
\end{tabular}

Values in bold face are statistically significant. SLE: systemic lupus erythematosus; CV: cardiovascular; AIC: Akaike information criterion; BIC: Schwarz Bayesian criterion; AUC: area under the curve; pfitHL: Hosmer-Lemeshow goodness-of-fit; SLICC: Systemic Lupus International Collaborating Clinics/American College of Rheumatology Damage Index; SLICC CV-: SLICC calculated without CV items.

\section{DISCUSSION}

The 2016 European Guidelines on CV disease prevention in clinical practice have established that documenting $\mathrm{CV}$ disease by invasive or noninvasive testing (such as carotid US to detect the presence of plaques) may be regarded as a risk modifier in CV risk prediction in some cases ${ }^{24}$. Therefore, although formal reclassification analyses have not been undertaken in the general population, carotid artery plaque assessment using US has gained support as a way to reclassify those patients for whom the SCORE is thought to have underestimated the true $\mathrm{CV}$ risk. To our knowledge, our study is the first to examine the effectiveness of carotid US in identifying high-risk patients with SLE for whom the CV risk had been previously assessed by means of SCORE risk charts.
Previous studies reported a higher frequency of carotid atherosclerotic plaques in patients with SLE than in controls ${ }^{25,26,27}$. In our analysis, $36 \%$ of patients with SLE were found to have either unilateral or bilateral carotid plaques. Additionally, traditional CV risk factors were found to be highly prevalent in our cohort. This is also in agreement with previous studies that showed an increased prevalence of traditional risk factors of atherosclerosis in patients with $\mathrm{SLE}^{28,29,30}$. Similarly, a series of 250 female patients with SLE from the Toronto Lupus Cohort showed higher prevalence of HTN, diabetes, premature menopause, and sedentary lifestyle than controls ${ }^{31}$.

In RA (the prototype of inflammatory joint disease), CV disease risk charts developed for the general population 
underestimated the proportion of patients at high risk for $\mathrm{CV}$ disease $^{32}$. Risk calculators recommended for patients with RA including the EULAR 1.5 multiplier, the Expanded CV Risk Prediction Score for RA, and QRISK2 did not predict $\mathrm{CV}$ disease risk more accurately than $\mathrm{CV}$ risk calculators developed for the general population ${ }^{32}$. This was also reported in patients with axial spondyloarthritis in whom the use of carotid US facilitated the identification of a high CV risk. Indeed, these patients had previously been classified as being at moderate $\mathrm{CV}$ risk when SCORE risk charts were applied $^{33}$.

Our study demonstrates that carotid US is also useful for identifying patients with SLE at high CV risk. In our series, only $6 \%$ of the patients met the definitions for high or very high risk when the SCORE risk charts were applied. The number of patients included in the categories of high or very high CV risk increased to $37 \%$ when carotid US was performed. Therefore, our findings support the use of carotid US to identify patients with SLE at high risk of CV disease.

We observed that age, HTN, and TGC serum levels were associated with an increased probability of patients with SLE being reclassified in the regression analysis. Moreover, disease-related data were also associated with an increased probability of patient reclassification. In fact, in addition to disease duration, we found that an SLICC $>0$ and C3 serum levels were factors that, after adjusting for traditional CV risk factors, were associated with a high risk of reclassification. Further, when we set up a predictive model on the probability of being reclassified, we found that disease-related factors such as disease duration, an SLICC CV-> 0 , and C3 serum levels when combined with age, HTN, TGC, and waist circumference, were capable of explaining such reclassification. This is of importance because these data show for the first time, to our knowledge, that $\mathrm{CV}$ risk reclassification of patients with SLE using carotid US can be attributed to the damage caused by the disease. These findings reinforce the concept that reclassification may not only be driven by the presence of conventional $\mathrm{CV}$ risk factors. That is, the disease itself or its interaction with genetic and traditional CV risk factors along with chronic inflammation, may constitute the key elements leading to the reclassification of patients with SLE into the very high CV-risk category.

Remarkably, in our study, complement serum levels were found to be positively associated with reclassification into the very high $\mathrm{CV}$-risk category. It is widely known that the cleavage of complement in tandem with the production of breakdown products is characteristic of most patients with active SLE. However, a similar positive association was found in a longitudinal study of patients with $\mathrm{SLE}^{34}$. In this study, C3 and C5a levels were identified as significant independent predictors of cIMT progression after 2 years of followup. Moreover, previous epidemiological studies showed that this complement system is associated with the development of atherosclerosis, while serum $\mathrm{C} 3$ and $\mathrm{C} 4$ levels are linked to an increased risk of $\mathrm{CV}$ disease $35,36,37,38,39$. For this reason, we believe that although SLE has been linked to complement system consumption, its positive association with atherosclerosis may be maintained.

It must be pointed out that comprehensive cardiometabolic evaluations remain poorly integrated into the management of patients with SLE, because of a limited awareness of the problem, lack of appropriate clinical studies, and poor strategies for CV risk reduction in SLE. For example, one study demonstrated how only $17 \%$ of patients with SLE believed that they were at high risk for developing coronary disease within 5 years, when in fact 3 or more traditional risk factors were present in 53\% of those who had a mean age of 38 years $^{40}$. For this reason, we believe that both patients with SLE and the clinicians who treat them should be aware of the importance of identifying high $\mathrm{CV}$-risk patients and making prevention of $\mathrm{CV}$ disease events a top priority. It is possible that assessments to determine the presence of carotid plaques could raise awareness of this problem in patients with SLE.

To our knowledge, our study is the first to examine how carotid US assessment permits the identification of patients with SLE at high CV risk who had previously been classified in categories of low and moderate $\mathrm{CV}$ risk when SCORE risk charts were used. In such individuals, disease damage seems to predict the presence of plaques, thereby facilitating the accurate reclassification of these patients into the very high CV-risk category.

\section{REFERENCES}

1. Asanuma Y, Oeser A, Shintani AK, Turner E, Olsen N, Fazio S, et al. Premature coronary-artery atherosclerosis in systemic lupus erythematosus. N Engl J Med 2003;349:2407-15.

2. Urowitz MB, Ibanez D, Gladman DD. Atherosclerotic vascular events in a single large lupus cohort: prevalence and risk factors. J Rheumatol 2007;34:70-5.

3. Urowitz MB, Gladman D, Ibanez D, Fortin P, Sanchez-Guerrero J, Bae S, et al; Systemic Lupus International Collaborating Clinics. Accumulation of coronary artery disease risk factors over three years: data from an international inception cohort. Arthritis Rheum 2008;59:176-80.

4. Karp I, Abrahamowicz M, Fortin PR, Pilote L, Neville C, Pineau $\mathrm{CA}$, et al. Recent corticosteroid use and recent disease activity: independent determinants of coronary heart disease risk factors in systemic lupus erythematosus? Arthritis Rheum 2008;59:169-75.

5. Magder LS, Petri M. Incidence of and risk factors for adverse cardiovascular events among patients with systemic lupus erythematosus. Am J Epidemiol 2012;176:708-19.

6. Abu-Shakra M, Urowitz MB, Gladman DD, Gough J. Mortality studies in systemic lupus erythematosus. Results from a single center. I. Causes of death. J Rheumatol 1995;22:1259-64.

7. Schoenfeld SR, Kasturi S, Costenbader KH. The epidemiology of atherosclerotic cardiovascular disease among patients with SLE: a systematic review. Semin Arthritis Rheum 2013;43:77-95.

8. Manzi S, Meilahn EN, Rairie JE, Conte CG, Medsger TA Jr., Jansen-McWilliams L, et al. Age-specific incidence rates of myocardial infarction and angina in women with systemic lupus erythematosus: comparison with the Framingham Study. Am J Epidemiol 1997;145:408-15.

9. D’Agostino RB Sr., Vasan RS, Pencina MJ, Wolf PA, Cobain M,

Personal non-commercial use only. The Journal of Rheumatology Copyright (C) 2019. All rights reserved. 
Massaro JM, et al. General cardiovascular risk profile for use in primary care: the Framingham Heart Study. Circulation 2008; 117:743-53.

10. Conroy RM, Pyorala K, Fitzgerald AP, Sans S, Menotti A, De Backer G, et al; SCORE project group. Estimation of ten-year risk of fatal cardiovascular disease in Europe: the SCORE project. Eur Heart J 2003;24:987-1003

11. Piepoli MF, Hoes AW, Agewall S, Albus C, Brotons C, Catapano AL, et al. 2016 European Guidelines on cardiovascular disease prevention in clinical practice: The Sixth Joint Task Force of the European Society of Cardiology and Other Societies on Cardiovascular Disease Prevention in Clinical Practice (constituted by representatives of 10 societies and by invited experts) Developed with the special contribution of the European Association for Cardiovascular Prevention \& Rehabilitation (EACPR). Atherosclerosis 2016;252:207-74.

12. Goldberg RJ, Urowitz MB, Ibanez D, Nikpour M, Gladman DD. Risk factors for development of coronary artery disease in women with systemic lupus erythematosus. J Rheumatol 2009;36:2454-61

13. Esdaile JM, Abrahamowicz M, Grodzicky T, Li Y, Panaritis C, du Berger R, et al. Traditional Framingham risk factors fail to fully account for accelerated atherosclerosis in systemic lupus erythematosus. Arthritis Rheum 2001;44:2331-7.

14. O'Neill SG, Pego-Reigosa JM, Hingorani AD, Bessant R, Isenberg DA, Rahman A. Use of a strategy based on calculated risk scores in managing cardiovascular risk factors in a large British cohort of patients with systemic lupus erythematosus. Rheumatology 2009;48:573-5.

15. Corrales A, Parra JA, González-Juanatey C, Rueda-Gotor J, Blanco $\mathrm{R}$, Llorca $\mathrm{J}$, et al. Cardiovascular risk stratification in rheumatic diseases: carotid ultrasound is more sensitive than Coronary Artery Calcification Score to detect subclinical atherosclerosis in patients with rheumatoid arthritis. Ann Rheum Dis 2013;72:1764-70.

16. Corrales A, Gonzalez-Juanatey C, Peiro ME, Blanco R, Llorca J, Gonzalez-Gay MA. Carotid ultrasound is useful for the cardiovascular risk stratification of patients with rheumatoid arthritis: results of a population-based study. Ann Rheum Dis 2014;73:722-7.

17. Agca R, Heslinga SC, Rollefstad S, Heslinga M, McInnes IB, Peters $\mathrm{MJ}$, et al. EULAR recommendations for cardiovascular disease risk management in patients with rheumatoid arthritis and other forms of inflammatory joint disorders: 2015/2016 update. Ann Rheum Dis 2017;76:17-28

18. Hochberg MC. Updating the American College of Rheumatology revised criteria for the classification of systemic lupus erythematosus. Arthritis Rheum 1997;40:1725.

19. Gladman DD, Ibanez D, Urowitz MB. Systemic lupus erythematosus disease activity index 2000. J Rheumatol 2002;29:288-91.

20. Gladman D, Ginzler E, Goldsmith C, Fortin P, Liang M, Urowitz M, et al. The development and initial validation of the Systemic Lupus International Collaborating Clinics/American College of Rheumatology damage index for systemic lupus erythematosus. Arthritis Rheum 1996;39:363-9.

21. Mosca M, Bombardieri S. Assessing remission in systemic lupus erythematosus. Clin Exp Rheumatol 2006;6 Suppl 43:S-99-104.

22. Katz JD, Senecal JL, Rivest C, Goulet JR, Rothfield N. A simple severity of disease index for systemic lupus erythematosus. Lupus 1993;2:119-23

23. Touboul PJ, Hennerici MG, Meairs S, Adams H, Amarenco P, Bornstein N, et al. Mannheim carotid intima-media thickness consensus (2004-2006). An update on behalf of the Advisory Board of the 3rd and 4th Watching the Risk Symposium, 13th and 15th European Stroke Conferences, Mannheim, Germany, 2004, and Brussels, Belgium, 2006. Cerebrovasc Dis 2007;23:75-80.
24. Piepoli MF, Hoes AW, Agewall S, Albus C, Brotons C, Catapano AL, et al; ESC Scientific Document Group. 2016 European Guidelines on cardiovascular disease prevention in clinical practice: The Sixth Joint Task Force of the European Society of Cardiology and Other Societies on Cardiovascular Disease Prevention in Clinical Practice (constituted by representatives of 10 societies and by invited experts) Developed with the special contribution of the European Association for Cardiovascular Prevention \& Rehabilitation (EACPR). Eur Heart J 2016;37:2315-81.

25. Roman MJ, Shanker BA, Davis A, Lockshin MD, Sammaritano L, Simantov R, et al. Prevalence and correlates of accelerated atherosclerosis in systemic lupus erythematosus. N Engl J Med 2003;349:2399-406.

26. Thompson T, Sutton-Tyrrell K, Wildman RP, Kao A, Fitzgerald SG, Shook B, et al. Progression of carotid intima-media thickness and plaque in women with systemic lupus erythematosus. Arthritis Rheum 2008;58:835-42.

27. Kao AH, Lertratanakul A, Elliott JR, Sattar A, Santelices L, Shaw P, et al. Relation of carotid intima-media thickness and plaque with incident cardiovascular events in women with systemic lupus erythematosus. Am J Cardiol 2013;112:1025-32.

28. Fernandez-Nebro A, Rua-Figueroa I, Lopez-Longo FJ, Galindo-Izquierdo M, Calvo-Alen J, Olive-Marques A, et al; EAS-SER (Systemic Diseases Study Group of Spanish Society of Rheumatology). Cardiovascular Events in Systemic Lupus Erythematosus: A Nationwide Study in Spain From the RELESSER Registry. Medicine 2015;94:e1183.

29. Zhang M, Qi C, Cao L, Qian J, Ni Z. Metabolic syndrome is correlated with carotid atherosclerosis in patients with lupus nephritis. Am J Med Sci 2014;348:486-91.

30. Sanchez-Perez H, Tejera-Segura B, de Vera-Gonzalez A, Gonzalez-Delgado A, Olmos JM, Hernandez JL, et al. Insulin resistance in systemic lupus erythematosus patients: contributing factors and relationship with subclinical atherosclerosis. Clin Exp Rheumatol 2017;35:885-92.

31. Bruce IN, Urowitz MB, Gladman DD, Ibanez D, Steiner G. Risk factors for coronary heart disease in women with systemic lupus erythematosus: the Toronto Risk Factor Study. Arthritis Rheum 2003;48:3159-67.

32. Crowson CS, Gabriel SE, Semb AG, van Riel PL, Karpouzas G, Dessein PH, et al; Trans-Atlantic Cardiovascular Consortium for Rheumatoid Arthritis. Rheumatoid arthritis-specific cardiovascular risk scores are not superior to general risk scores: a validation analysis of patients from seven countries. Rheumatology 2017;56:1102-10.

33. Rueda-Gotor J, Llorca J, Corrales A, Parra JA, Portilla V, Genre F, et al. Cardiovascular risk stratification in axial spondyloarthritis: carotid ultrasound is more sensitive than coronary artery calcification score to detect high-cardiovascular risk axial spondyloarthritis patients. Clin Exp Rheumatol 2018;36:73-80.

34. Rua-Figueroa I, Arencibia-Mireles O, Elvira M, Erausquin C, Ojeda $\mathrm{S}$, Francisco F, et al. Factors involved in the progress of preclinical atherosclerosis associated with systemic lupus erythematosus: a 2-year longitudinal study. Ann Rheum Dis 2010;69:1136-9.

35. Capuano V, D'Arminio T, La Sala G, Mazzotta G. The third component of the complement (C3) is a marker of the risk of atherogenesis. Eur J Cardiovasc Prev Rehabil 2006;13:658-60.

36. Engstrom G, Hedblad B, Janzon L, Lindgarde F. Complement C3 and $\mathrm{C} 4$ in plasma and incidence of myocardial infarction and stroke: a population-based cohort study. Eur J Cardiovasc Prev Rehabil 2007;14:392-7.

37. van Greevenbroek MM, Jacobs M, van der Kallen CJ, Vermeulen VM, Jansen EH, Schalkwijk CG, et al. The cross-sectional association between insulin resistance and circulating complement C3 is partly explained by plasma alanine aminotransferase, 
independent of central obesity and general inflammation (the CODAM study). Eur J Clin Invest 2011;41:372-9.

38. Wlazlo N, van Greevenbroek MM, Ferreira I, Jansen EJ, Feskens EJ, van der Kallen CJ, et al. Low-grade inflammation and insulin resistance independently explain substantial parts of the association between body fat and serum C3: the CODAM study. Metabolism 2012;61:1787-96.
39. Nilsson B, Hamad OA, Ahlstrom H, Kullberg J, Johansson L, Lindhagen L, et al. C3 and C4 are strongly related to adipose tissue variables and cardiovascular risk factors. Eur J Clin Invest 2014;44:587-96.

40. Petri M, Spence D, Bone LR, Hochberg MC. Coronary artery disease risk factors in the Johns Hopkins Lupus Cohort: prevalence, recognition by patients, and preventive practices. Medicine 1992;71:291-302 